Conclusion In patients with STIs/HIV, when using syndromic approach nearly half of the male patients had uretharal discharge syndrome and over half of female patients had vaginal discharge syndrome. Etiologically, genital warts due to HPV were the most common cause.

\section{P2.137 SYNDROMIC MANAGEMENT OF VAGINAL DISCHARGE: IS IT JUSTIFIED?}

doi:10.1136/sextrans-2013-051184.0401

\begin{abstract}
'P Mittal, ${ }^{2}$ S C Sonkar, ${ }^{3} \mathrm{P}$ K Mishra, ${ }^{1} \mathrm{~J}$ Suri, , $\mathrm{A}$ Kumar, ${ }^{1} \mathrm{P}$ Ghope, ${ }^{4} \mathrm{D}$ Saluja. ${ }^{1} \mathrm{~V} M$ Medical college \& Safdarjung Hospital, Delhi, India; ${ }^{2} \operatorname{Dr}$ B.R. Ambedkar Center for Biomedical Research, university of Delhi, India; ${ }^{3} \mathrm{Dr}$ B.R. Ambedkar Center for Biomedical Research, University of Delhi, India; ${ }^{4} \mathrm{Dr}$ B.R. Ambedkar Center for Biomedical Research, university of Delhi, India
\end{abstract}

Introduction Vaginal discharge constitutes an important symptom in women attending gynaecology outpatient clinics. Some of these cases are due to sexually transmitted organisms such as $\mathrm{N}$. gonorrheae, C. trachomatis and $\mathrm{T}$. vaginalis and can lead to poor reproductive health and co-infection with HIV. The control of these infections can lead to substantial reduction in the transmission of HIV. Given the high cost of diagnosis, National Aids Control Organisation advocates syndromic management of patients with genitourinary complaints and treatment with drugs that target the most frequent etiological agents. However, it can lead to emergence of antibiotic resistant strains due to overtreatment. Therefore, we tried to find out prevalence of these three pathogens in cases of symptomatic vaginal discharge and to determine the number of patients who were over-treated following syndromic approach.

Methods 320 non-pregnant women (16 - 60 years) attending the gynaecology outpatient clinic of VMMC \& Safdarjung Hospital, New Delhi, with complaint of vaginal discharge and clinically suspected to be infected by N. gonorrhoeae, C. trachomatis \& T. Vaginali were recruited. Endocervical swabs were used for detection of these organisms by PCR.

Results Of the 320 subjects 24 (7.5\%) were positive for C. trachomatis, 19 (5.9\%) for N. gonorrhoeae \& 13 (4.6\%) for T. vaginalis. Furthermore, $8(2.5 \%)$ patients had co-infection of C. trachomatis \& $\mathrm{N}$. gonorrhoeae, $07(2.1 \%)$ had co-infection of C. trachomatis \& $\mathrm{T}$. vaginalis, $07(2.1 \%)$ had $\mathrm{N}$. gonorrheae \& $\mathrm{T}$. vaginalis and $06(1.8 \%)$ cases had C. trachomatis, N. gonorrheae \& T. vaginalis. Infection was found in $49 / 320$ patients with infection rate of $15.3 \%$. Since all the patients (320) were treated the overtreatment rate was $84.6 \%$.

Conclusion Cost-effective technology for simultaneous detection of these pathogens is urgently required in developing countries so that all clinically suspicious cases of vaginal discharge are given treatment only after confirmed diagnosis.

\section{P2.138 CONGENITAL SYPHILIS IN A NEWBORN}

doi:10.1136/sextrans-2013-051184.0402

${ }^{1} \mathbf{K}$ Chudomirova, ${ }^{2} \mathrm{~T}$ Shmilev, ${ }^{2} \mathrm{M}$ Panova. 'Medical University, Department of dermatology and venereology, Plovdiv, Bulgaria; ${ }^{2}$ Medical University, Department of paediatrics, Plovdiv, Bulgaria

Objectives Although congenital syphilis is a completely preventable disease, its incidence in Bulgaria has experienced an increase in the recent 15 years.

Methods A 25-day male newborn with a wide spectrum of clinical features of congenital syphilis is presented.

Results The child was hospitalised in a quite bad condition with fever, interstitial pneumonia, swollen and painful knee and tarsal joints, reduced active movement of the left schoulder, and erythemo-bullous rush of the palms and soles (palmoplantar pemphigus). The bone radiogram showed features of osteochondritis and periostitis of the femurs. The laboratory studies revealed haematological abnormalities (anaemia, leucocytosis), signs of inflammation and increase in aminotransferases. The syphilis blood serology was positive. The mother did not attend for antenatal care during her pregnancy and early latent syphilis was diagnosed. The treatment with Penicillin $G$ led to a rapid improvement of the clinical status of the newborn.

Conclusions This case stresses on the variety of clinical symptoms and the main factors contributing to the raise of the congenital syphilis, i.e. insufficient prenatal care and reduced or absent screening in pregnant mothers, suboptimal laboratory diagnosis, and lack of sexual knowledge especially among the vulnerable groups of the community. The need of optimal syphilis management is obviously.

\section{P2.139 SYSTEMATIC REVIEW: SYNDROMIC MANAGEMENT OF VAGINAL DISCHARGE FOR TREATMENT OF GONORRHOEA AND CHLAMYDIA}

doi:10.1136/sextrans-2013-051184.0403

${ }^{1}$ G Hovhannisyan, ${ }^{1} \mathrm{~A} J$ Rotstein, ${ }^{2} \mathrm{C}$ Lee, ${ }^{3} \mathrm{~V}$ Allen, ${ }^{3} \mathrm{~S}$ Bondy. ${ }^{1} \mathrm{McM}$ aster University, Hamilton, ON, Canada; ${ }^{2}$ Public Health Ontario, Toronto, ON, Canada; ${ }^{3}$ University of Toronto, Toronto, ON, Canada

Background Syndromic management of vaginal discharge is common in both developed and developing countries. No systematic review has evaluated the performance of the WHO syndromic management algorithms as a case-management tool for symptomatic women. Our evaluation will inform health care professionals whether these algorithms have sufficient discriminatory power in identifying patients who would benefit from treatment for cervical infections.

Methods We conducted a systematic review and meta-analysis of diagnostic studies on syndromic management of vaginal discharge. We searched Medline, Embase and Global Health databases; we also searched relevant lists of references. We included the studies that reported their findings in sufficient detail to allow data abstraction for symptomatic women. Sensitivity, specificity and diagnostic OR (DOR) were used to evaluate the algorithms. ROC curves were pooled using the HSROC random effects model.

Findings We screened 896 abstracts for eligibility and included 101 articles for a full text review; 17 studies were included in the analysis. HSROC overall summary statistics were: sensitivity $0.58(0.42$ $0.73)$, specificity $0.70(0.61-0.78)$, DOR $3.25(2.22-4.76)$ and sensitivity 0.81 (0.71-0.87), specificity $0.46(0.40-0.52)$, DOR 3.71 (2.42-5.67) for the WHO algorithms without and with speculum examination respectively. For the algorithms that were modified to include local risk factors the overall sensitivity was 0.75 (0.51-0.90), specificity $0.58(0.35-0.77)$ and DOR 4.18 (2.84-6.15) for the algorithms without speculum exam; sensitivity 0.70 (0.41-0.89), specificity 0.64 (0.42-0.82), DOR 4.24 (2.55-7.54) for the algorithms with speculum exam; and sensitivity 0.91 (0.68-0.98), specificity 0.54 (0.31-0.75), DOR 11.97 (4.93-30.0) for the algorithms with microscopy.

Interpretation Overall, diagnostic algorithms for the management of vaginal discharge had poor to moderate performance in symptomatic women. Incorporating locally-identified risk factors resulted in small improvement in the performance of the algorithms, however, considerable number of women were overtreated due to low specificity.

\section{P2.140 TIME TRENDS OF C. TRACHOMATIS SEROTYPE DISTRIBUTIONS IN FERTILE-AGED WOMEN IN FINLAND}

doi:10.1136/sextrans-2013-051184.0404

J Paavonen. Department of Obstetrics and Gynecology, University Hospital, Helsinki, Finland 
The National Institute of Health and Welfare, Oulu, Universities of Oulu, Tampere and Helsinki, Finland.

We studied C. trachomatis (CT) serotype-specific antibodies in Finnish women between 1980's and 2000's. C. trachomatis seropositive women $(1,169)$ were available from a subcohort $(11,067)$ of the Finnish Maternity Cohort representing the 1980's $(\mathrm{N}=480)$ and 2000 's ( $\mathrm{N}=331$ ) for serotype specific classification by microimmunofluorescence test. Serotype distributions were comparable in the 1980's and 2000's, G, E and J being the most prevalent. Serotype D peaked in the 1990's. The overall serotype profile changed first between 1980's and 1990's but changed back in the 2000's. The rates of women with antibodies against two or more serotypes increased in the 1990's, but then decreased again. In conclusion, we analysed the rates and distributions of $C T$ serotype-specific antibodies from the 1980's to 2000's in fertile-aged female population in Finland. We found that CT serotype distributions varied considerably over time.

\section{P2.141 KNOWLEDGE, ATTITUDES AND PRACTICES OF HEALTHCARE WORKERS FROM UNIVERSITY HOSPITAL OF OUAGADOUGOU (BURKINA FASO) FACE TO ACCIDENTAL BLOOD EXPOSURE (ABE) AND BIOLOGICAL FLUIDS IN CAREGIVING FACILITIES}

doi:10.1136/sextrans-2013-051184.0405

'Y Sagna, ${ }^{2} \mathrm{~S}$ Kouanda, ${ }^{1} \mathrm{H}$ Tiéno, ${ }^{1} \mathrm{R}$ A K Nianogo, ${ }^{1} \mathrm{R}$ Bognounou, ${ }^{1} \mathrm{~J}$ Y Drabo. 'Internal Medicine Department of Yalgado Ouédraogo University Hospital (CHU YO), Ouagadougou, Burkina Faso; 'Institut de recherche en sciences de la santé, Ouagadougou, Burkina Faso

Objective Accidental blood exposure $(\mathrm{ABE})$ is defined as "any accidental exposure to blood or to a biological fluid contaminated by blood, including a cutaneous injury during an incision or injection, or a projection on mucosa or on an injured skin". we aimed to evaluate the knowledge, attitudes and practises of healthcare workers in one of West Africa country national referral hospital (Yalgado Ouédraogo University Hospital) face to ABE.

Methods A descriptive cross-sectional and analytical study was conducted, from May to July 2009, on all healthcare workers (medical, paramedical and support staff) working for at least a year in this hospital university. A self and anonymous questionnaire was used to gather the information. The knowledge assessment was made using a digital scale. Results 462 healthcare workers responded to the questionnaire (response rate: $51.3 \%$ ). They composed of 60 physicians $(12.9 \%)$, 313 paramedics $(67.7 \%)$ and 89 support officers $(19.2 \%)$. The exact definition of an $\mathrm{ABE}$ was known from 255 healthcare workers $(55.2 \%)$ and $375(81.2 \%)$ were aware of the principles of universal precautions. Medical staff better defined the ABE than the other healthcare workers $(p=0.00) .133$ healthcare workers $(29.4 \%)$ were at least once a victim of an ABE, of which 84 (61.3\%) had not reported their $\mathrm{ABE}$. Ignorance of the support procedures was in $26.8 \%$ of cases the main cause of no report. The risk of contamination after $\mathrm{ABE}$ had not been assessed in $60.3 \%$ of cases. Only $69.7 \%$ of the healthcare workers were aware that HIV is a potentially transmissible infectious agent during an $\mathrm{ABE}$. Protective equipment was not always available and not constantly used.

Conclusion The ABE knowledge of these referral hospital healthcare workers is not satisfactory. Appropriate communication and awareness means on the risks associated with the ABE should be further developed in healthcare settings.

\section{P2.142 T00 BAD! SINGLE MOLECULE ANTIRETROVIRAL DRUGS MAY NOT BE THE MAGIC BULLET}

doi:10.1136/sextrans-2013-051184.0406

'T Agyarko-Poku, ${ }^{2} Y$ Adu Sarkodie. 'Suntreso Government Hospital, Ghana Health Services, Kumasi, Ghana; ${ }^{2}$ School of Medical Sciences, Kwame Nkrumah University of Science and Technology, Kumasi, Ghana
Background Adherence to Antiretroviral drugs (ARVs) poses a challenge for an effective Antiretroviral Therapy in Ghana. The introduction of single molecule ARVs regime was seen as an antidote to non-adherence among HIV patients on treatment. This study was conducted among ARVs experienced patients, who were about to be switched onto single molecules, to determine their perception on the new treatment regime.

Methods 1681 HIV positive patients from Suntreso STI/HIV Clinic in Kumasi, who have been on treatment for more than 12 months and consented to participate in this cross sectional study, were interviewed using structured pretexted questionnaire prior to the commencement of the new single molecule ARVs treatment regime. Data was entered and analysed using SPSS version 16.

Results Whereas $60.9 \%(1023 / 1681)$ find the present dose regime cumbersome, $39.1 \%(657 / 1681)$ prefer the multiple dose regime. $42.2 \%$ (709) of respondents have some reservations about single dose regime whilst $24.6 \%$ (413) prefer it with $33.3 \%(559)$ being indifferent. Reasons for the reservation included; 'Side effect may be too serious' (48.3\%, 342/709), 'Virus too powerful for a single molecule' $(30.2 \%, 214 / 709)$, 'Attempts to deprive us of drugs and facilitate our death' $(13.8 \%, 92 / 709)$ and 'Cost of drugs will be expensive in future for a combine drugs' $(7.7 \%, 55 / 709)$.

Conclusion Although majority of patients find the multiple dose regimes cumbersome, they are sceptical about the use of the single molecule regime. The new regime may result in overdosing if they find it inadequate to provide the needed protection. The fear of serious adverse reaction from combination of ARVs compared with separate drugs, may scare them from taking the treatment. Intensive adherence counselling taking care of the above concerns is essential before patients are switch onto the single molecule ARV regime

\section{P2.143 BENEFITS, CHALLENGES AND LESSONS LEARNED IN IMPLEMENTING AN HIV MEDICATION DELIVERY SCHEME}

doi:10.1136/sextrans-2013-051184.0407

L Hilton, K A Fernando, C Penn, H Jaleel, J H Day. Department of Sexual Health \& HIV Medicine, Southend University Hospital NHS Foundation Trust, Essex, UK

Introduction The HIV department of Southend University Hospital NHS Foundation Trust UK, care for 370 patients of which $80 \%$ are taking antiretroviral therapy(ART).

Monthly ART spend is approximately $£ 200,000$. A proposal for ART home delivery was made by the HIV multidisciplinary team(MDT) and local procurement pharmacists. The desired outcome being patient convenience, whilst making financial savings for the Trust in view of $0 \%$ value added tax for delivered medication.

Methods A delivery scheme began in June 2011. 60\% of patients on ART chose to enter it. 3 months later, however, failed deliveries were reported. This continued despite efforts to rectify issues with the delivery provider.

In November 2011, an alternative provider, with greater experience in ART, was recruited. Now, 15 months later, the scheme continues successfully, with $55 \%$ of patients on ART entered.

Outcomes: Initial patient survey identified potential concerns regarding entering the scheme - firstly, reliability of timing of medication delivery and secondly, protection of patient identifiable information.

The convenience of not waiting for ART to be dispensed in the hospital pharmacy is a key patient reported benefit. From the hospital perspective, home delivery is resulting in savings of $£ 9300$ per month. Additionally, there is less pharmacy storage space consumption.

Additional administration time consumption, however, is noted in 'trouble-shooting' and coordinating the process. 